\title{
New 4-D Imaging for Real-Time Intraoperative MRI: Adaptive 4-D Scan
}

\author{
Junichi Tokuda ${ }^{1}$, Shigehiro Morikawa ${ }^{2}$, Hasnine A. Haque ${ }^{3}$, \\ Tetsuji Tsukamoto ${ }^{3}$, Kiyoshi Matsumiya ${ }^{1}$, Hongen Liao ${ }^{4}$, Ken Masamune ${ }^{1}$, \\ and Takeyoshi Dohi ${ }^{1}$ \\ ${ }^{1}$ Graduate School of Information Science and Technology, The University of Tokyo, \\ 7-3-1, Hongo, Bunkyo, Tokyo 113-8656, Japan \\ junichi@atre.t.u-tokyo.ac.jp \\ ${ }^{2}$ Biomedical MR Science Center, Shiga University of Medical Science, \\ Seta-tsukinowa Cho, Otsu, Shiga 520-2192, Japan \\ ${ }^{3}$ GE Yokogawa Medical Systems Ltd., \\ 4-7-127, Asahigaoka, Hino, Tokyo 191-8503, Japan \\ ${ }^{4}$ Graduate School of Engineering, The University of Tokyo, \\ 7-3-1, Hongo, Bunkyo, Tokyo 113-8656, Japan
}

\begin{abstract}
Aiming at real-time 3-D visualization of organ motion to navigate surgical procedures in MRI-guided surgery, a new 4-D MR imaging technique called "Adaptive 4-D Scan" has been proposed. The technique is designed to acquire a time series of volumetric 3-D images (4-D image) of cyclically moving organ, even in a low-field open-configuration MR scanner. A pre-operative 4-D image is acquired with respiratory phase parameter, which is monitored by using navigator-echo-based realtime tracking of the liver and diaphragm. During operation, the respiratory phase is again monitored in real-time, and a 3-D image, reflecting the current state of the target organ, is extracted from the pre-operative 4-D image and provided to physicians as a pseudo real-time 3-D image. We implemented Adaptive 4-D Scan into a 0.5 Tesla open-configuration clinical MRI system for intervention. Phantom and volunteer studies were performed to assess feasibility of this technique, in terms of image quality, imaging time and position accuracy of the imaged subject. A 4-D image (matrix: $256 \times 128 \times 10 \times 8$ ) of cyclically moving phantom was acquired in $719 \mathrm{~s}$, and RMS position error between the imaged subject and the real subject was $2.3 \mathrm{~mm}$, where the range of motion was 50 mm. 4-D image of the moving liver was also successfully acquired under near clinical condition. In conclusion, the study shows that the proposed method is feasible and has capability to provide real-time dynamic 3-D atlas for surgical navigation.
\end{abstract}

\section{Introduction}

Intraoperative Magnetic Resonance Imaging (MRI) has been recognized as a promising tool for surgical guidance in recent years. With the introduction of open-configuration MRI, variety of surgical application has been explored to investigate the benefit of intraoperative imaging with soft tissue discrimination. 
Despite the merit of accessible MRI in surgery, its limited applicability to moving organs is unsolved issue. Generally, it takes more than a few seconds to acquire even a slice of MR image; therefore an image inherently has misalignment to the actual organ when the images are provided to the physician. Furthermore, fast motion of the target causes serious artifact on a MR image, which makes it difficult for physicians to discriminate the lesion and make accurate decision in targeting. These are the major issues in intraoperative MRI before expanding its application target to moving organ in abdominal cavity, and have been discussed frequently in the community [1]-3].

To capture an image of organs moving due to respiration, several techniques have been proposed. Liu et. al. proposed navigator gating technique [4, which enables to reduce motion artifact by accepting MR echo signal data only when the respiratory state is in the certain range (gating window). Although the method has been widely used for image diagnosis of abdominal organs, it is not suitable for surgical guidance on moving organ, because images provide only static information at constant respiratory state. As an extended technique of navigator gating, Kolmogorov demonstrated multiple volume acquisition [5]. This technique allows us to acquire multiple images at different respiratory states, but number of gating windows, which equals to number of respiratory states, is determined by histogram of motion, and thus it is impossible to visualize full range of motion of the subject. Another approach is $4 \mathrm{D}$ MR imaging using internal respiratory gating proposed by Siebenthal et. al 3 . In this study, the $3 \mathrm{D}$ images of the liver were reconstructed for complete respiratory cycle by retrospective stacking of dynamic $2 \mathrm{D}$ images using internal image-based gating. Unfortunately, since the proposed method is based on fast 2-D imaging (in case of their study, they use sequence with frame rate of $175 \mathrm{~ms} /$ frame), the type of imaging sequence that can be used in this method is limited. Furthermore, a low-field open-configuration MR scanner, which is thought to be an ideal guidance tool for surgery, usually doesn't have capability to drive such a fast imaging sequence.

Based on these backgrounds, we propose a new 4-D MR imaging method, called Adaptive 4-D Scan, which can be implemented to a low-field open-configuration MR scanner, and is applicable for real-time 3-D surgical navigation. The ultimate goal of this work is to generate pseudo real-time 3-D image during operation, through following steps: (Step 1) a time series of 3-D images of a target organ covering whole respiratory cycle is acquired before operation using proposed method, (Step 2) a real-time tracking of the target organ performed during operation to detect respiratory phase of a patient, and (Step 3) best-fitted 3-D image is selected at each moment during operation based on the real-time respiratory phase parameter, and a pseudo real-time 3-D image is provided to physicians.

In this paper, we evaluated 4-D images in terms of image quality as well as position error between actual subject and imaged subject. To the authors' knowledge, none of the related work have argued image position error with a scope to guide surgery. However this is important issue for real-time surgical guidance, since the position information is used to guide surgical incision 


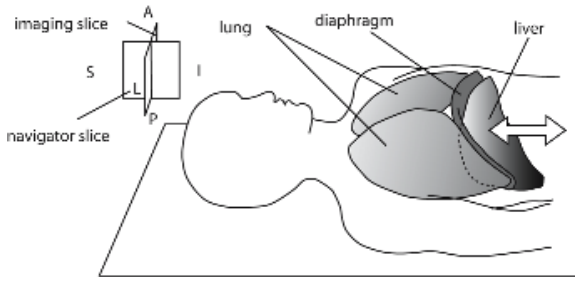

Fig. 1. To monitor respiratory phase of the patient, the shift of the liver was detected by acquiring navigator echoes from the sagittal slice. The liver mainly shifts in S-I direction with diaphragm during breath. Images were acquired from axial slices.

e.g. needle insertion, ablation. In addition, the liver of healthy volunteer was scanned with proposed method to assess feasibility on near clinical conditions.

\section{Materials and Methods}

Adaptive 4-D Scan technique is similar to respiratory gating, which enables to acquire single 3-D image of cyclically moving subject. The major difference between these two methods is adaptive update of scan parameters based on real-time feedback of respiratory phase to the pulse sequencer.

In this section, we describe two key techniques to implement Adaptive 4-D Scan: real-time respiratory monitoring to detect respiratory phase during scan, and adaptive control of scan parameters for 4-D imaging.

\subsection{Real-Time Respiratory Monitoring Using MR}

The basic principle of real-time respiratory monitoring is based on the navigator echo technique, originally proposed by Ehman 6 . A navigator echo is a magnetic resonance echo to detect the motion of the subject during image acquisition. Since only one or a few navigator echoes are required to detect the motion, time-resolution of motion tracking is much higher even than 2-D MR imaging.

In this study, we employed a navigator echo to detect the respiratory phase of the subject in real-time, by tracking shift of the liver, that moves mostly along superior-to-inferior axis during respiration as shown in Fig. 1. To acquire imaging echoes and navigator echoes simultaneously, we configured a pulse sequence, in which navigator echo acquisitions are inserted into repetition of imaging echo acquisitions. $N_{\text {intv }}$, number of imaging echo acquisitions between navigator echo acquisitions, is configurable. Frame rate of respiratory monitoring is determined as $\frac{1}{T R \times N_{\text {intv }}}$ fps, where $\mathrm{TR}$ is repetition time of $\mathrm{MR}$ echo acquisitions. In this study, we set $N_{\text {intv }}=5$.

\subsection{Adaptive Control of Scan Parameters for 4-D Scan}

To reconstruct a 4 -D image, $k_{x} \times k_{y} \times z \times t$ matrix needs to be filled up with MR echo signals $S\left(k_{x}, k_{y}, z, t\right)$, where $k_{x}$ and $k_{y}$ are frequency and phase encoding parameters for each, $z$ is the slice offset and $t$ is time. $t$ can be replaced by a respiratory phase parameter $\phi$, since we assume that motion of the target organ is cyclic. We note the number of steps of $\phi$ as $N_{\phi}$, which equals to image 
resolution in time dimension. In contrast to normal 3-D imaging, where $k_{y}$ and $z$ can be determined prior to start of a scan, the scan parameters are dynamically updated based on the respiratory phase $\phi$ of the subject in Adaptive 4-D Scan. This is because $\phi$ reflects the state of patient's free breath, and its variation is unpredictable before the scan.

To obtain $\phi$, we detect the shift of the liver and diaphragm in superior-toinferior direction by using navigator echo. In this study, $\phi$ was defined as $\phi=\frac{s}{L} \pi$, where $s$ is the shift of the liver and diaphragm from the reference point, and $L$ is maximum stroke of $s$. Note that the reference point is at the end of the stroke, and thus the range of $s$ is $0 \geq s \geq L$.

The example pseudocode for adaptive control of scan parameters would be:

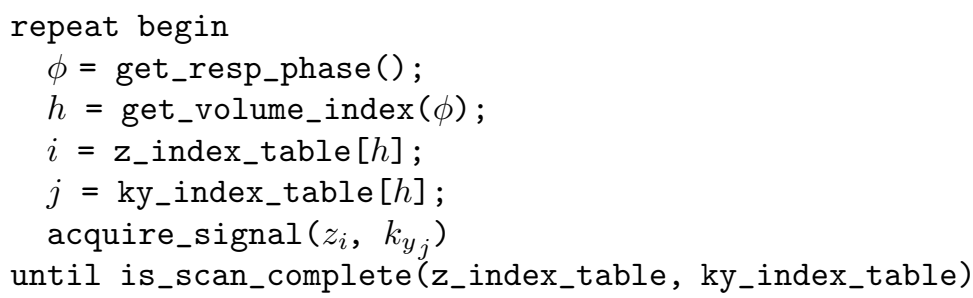

where $z_{-}$index_table [] and ky_index_table [] are tables to preserve $z$ and $k_{y}$ for each volume indexed by $h$. In procedure get_resp_phase (), a pulse sequence to acquire navigator echo is executed, and $\phi$ is returned. get_volume_index() converts $\phi$ to the volume index $h$. To convert $\phi$, which is a continuous value, to the index $h$, the range of variation of $\phi$ is split into $N_{\phi}$ partitions (Fig. 22). The width of each partition is determined in two ways: (A) $\Delta \phi_{h}=$ const. and (B) $T_{h}=$ const. for $h=1,2, \ldots, N_{\phi}$, where $\Delta \phi_{h}$ is width of each partition of $\phi$ and $T_{h}$ is total duration while $\phi$ is in $h^{\text {th }}$ partition. The merit using (B) is that echo signals are distributed to each partition evenly, and therefore imaging time can be reduced without compromising $N_{\phi}$. We implemented this method to an open-configuration MR system, GE Signa SP/i 0.5 T (GE Healthcare, Chalfont St. Giles, UK).

\section{Experiments and Results}

\subsection{Phantom Study}

We conducted the phantom study to evaluate image quality, imaging time and position accuracy of 4-D images, which are important issues for image guidance.

To simulate motion of a human liver, a cylindrical agar phantom was placed on a specially designed stage, which generated reciprocating motion with stroke of $L=50 \mathrm{~mm}$ (Fig. 3). The crank was rotated manually with speed of approx. 0.2 cycles per second.

In this study, the phase of the cyclical motion $\phi$ was determined by the shift of the phantom itself. To monitor $\phi$, the navigator echoes were acquired from a 


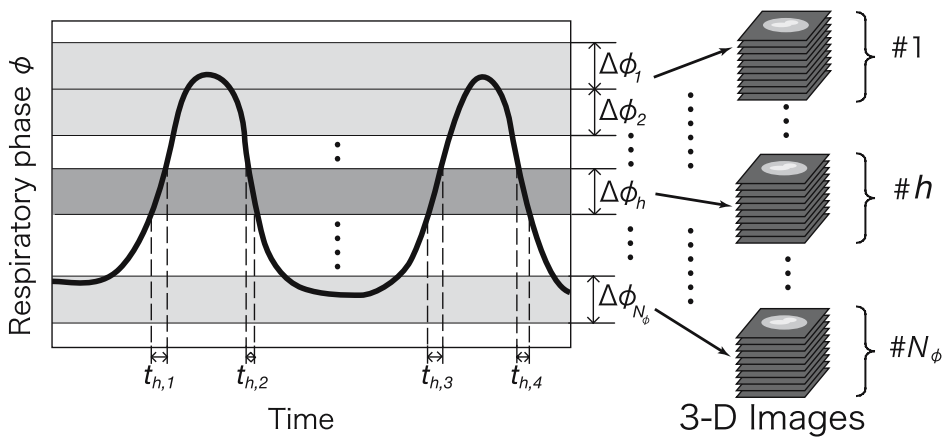

Fig. 2. The range of respiratory phase variation is split into $N_{\phi}$ partitions. Two methods to determine width of each partition were implemented: (A) $\Delta \phi_{1}=\Delta \phi_{2}=$ $\ldots=\Delta \phi_{N_{\phi}}$, where $\Delta \phi_{h}$ is width of the $h^{\text {th }}$ partition. (B) $T_{1}=T_{2}=\ldots=T_{N_{\phi}}$, where $h^{\text {th }}$ is amount duration while $\phi$ is in the $h^{\text {th }}$ partition. $T_{h}$ is defined by $T_{2}=t_{2,1}+t_{2,2}+t_{2,3}+t_{2,4}+\ldots$ in the graph.

plane parallel to the motion of the phantom. Imaging planes were also in the same direction, to measure the shift of the phantom from each 2-D slice image in $3-\mathrm{D}$ images independently. Consequently, the sample number of the measurements for one 4-D image equals to the number of slices.

The pulse sequence was based on fast gradient echo (FGRE), with parameters: TR:30 ms, TE:9.7 ms, flip angle:30 degrees and FOV:300 $\times 300 \mathrm{~mm}$. The matrix size of 4 -D images were $256 \times 128 \times 10 \times N_{\phi}$. 4-D images were acquired with $N_{\phi}=4,6,8,10$ using partition method (A), and with $N_{\phi}=6$ using partition method (B). Scans were performed twice for each condition.

We assessed imaging time, image quality and position error of imaged phantom for each 4-D image. "Ground-truth" for error evaluation was calculated based on the translation of the table. The table position was calibrated to the coordinate of the scanner. Since each 3-D image was reconstructed from MR echo signals acquired with different $\phi$, distributed within the corresponding phase partition, we defined "ground-truth" position for each 3-D image at the center of each partition. "Ground-truth" shift of the phantom from the base position (at the end of the stroke) in $h^{\text {th }}$ image can be calculated by $s_{h}=\frac{L}{\pi}\left(\frac{\Delta \phi_{h}}{2}+\sum_{k=1}^{h-1} \Delta \phi_{k}\right)$.

The part of result 4-D images for $N_{\phi}=4,6,8$ and 10 acquired using partition method (A) are shown in Fig 4, and the root mean square (RMS) error of phantom position and imaging time are shown in Fig. [5] (1). We found that there are trade-offs between image quality and imaging time, and between image position accuracy and imaging time except the case $N_{\phi}=10$.

In addition, the images acquired using partition method $(\mathrm{A})$ and $(\mathrm{B})$ are compared fixing $N_{\phi}$ at 6 . Fig 5 (2) shows the relation between image position error and distance from the center of the stroke. In both cases, position errors are higher at the centers of the stroke than at the ends of the stroke, but difference between minimum and maximum errors is much larger in method (A). The 


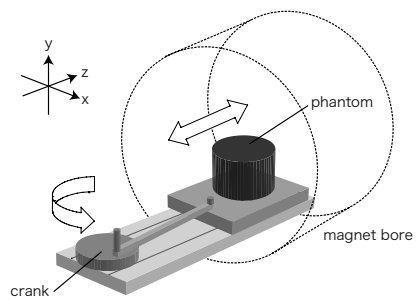

Fig. 3. The phantom was placed on the stage with a crank mechanism to make reciprocating motion with stroke of $50 \mathrm{~mm}$. The motion of the stage was limited along only one direction.

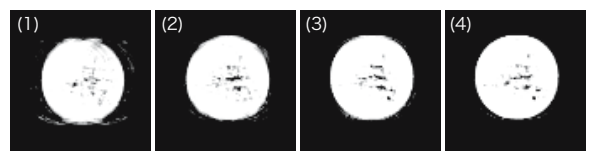

Fig. 4. 2-D images as part of result 4-D images are shown: (1) $N_{\phi}=4,(2) N_{\phi}=$ 6, (3) $N_{\phi}=8$ and (4) $N_{\phi}=10$. Image quality was improved when $N_{\phi}$ was set to larger value.

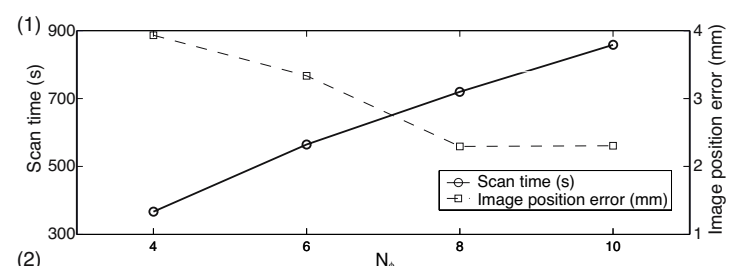

(2)

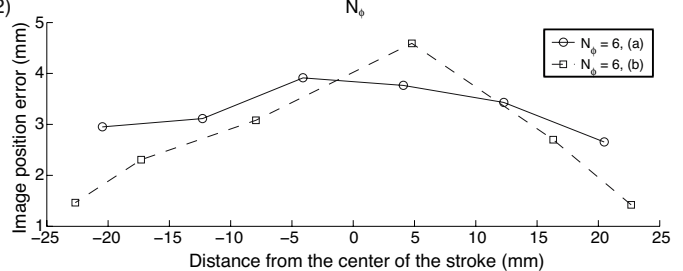

Fig. 5. Errors and time evaluation of the phantom study. RMS position errors and imaging time for $N_{\phi}=4,6,8$ and 10 fixing partition method to (A) are shown in (1). Also relation between errors and position of the phantom in method (A) and (B) are compared fixing $N_{\phi}$ in (2). Note that two 4-D images containing 10 slices were acquired for each condition, and errors were evaluated for each slice independently. Therefore the number of samples to calculate RMS error is 20. Imaging times are average time of two trials.

imaging time for $(\mathrm{A})$ and $(\mathrm{B})$ were $565 \mathrm{~s}$ and $472 \mathrm{~s}$, and time reduction by 16 percents was achieved.

\subsection{Volunteer Study}

To evaluate feasibility of Adaptive 4-D Scan, we performed study on the volunteer's liver. 4-D image of the liver of the healthy volunteer was scanned on near clinical conditions, while the volunteer was freely breathing. Adaptive 4-D Scan was performed with $N_{\phi}=5$, and number of slices of 21, using partition method (A). The pulse sequence was FGRE (TR:28 ms, TE:8.7 ms and flip angle:30 degrees). The scan slices were axial for imaging.

The 4-D image of the liver was successfully acquired in $1807 \mathrm{~s}$, and the part of the 4-D image is shown in Fig $6(1)$. The stroke of diaphragm shift in S-I direction was $13.9 \mathrm{~mm}$. The liver in each 3 -D volume was manually segmented and a surface model was generated (Fig. 6(2)). 

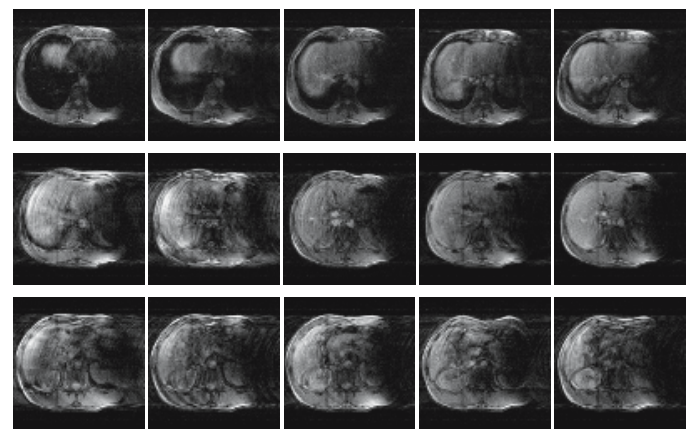

(1)

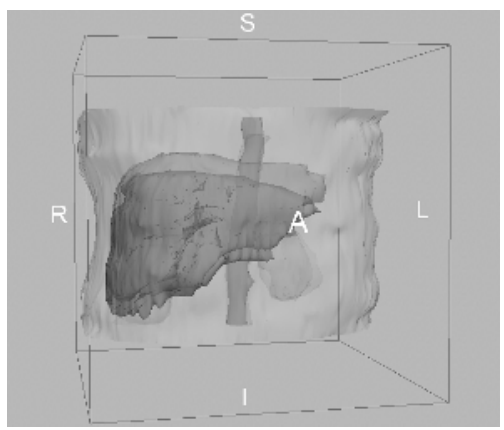

(2)

Fig. 6. 4-D image sized $256 \times 128 \times 21 \times 5$ pixels was acquired, in the volunteer study. As a part of the 4-D image, three slices of volunteer's liver at five different respiratory phases are shown in (1). Organs, vascular and other atlas can be discriminated. 3-D surface models of the liver at two different respiratory phases were created from the 4-D image, visualized on the surgical navigation software, 3D Slicer [7] in (2). The skin, aorta and kidneys are also visualized as position references.

\section{Discussion}

We demonstrated the new method to acquire 4-D image of moving organ with a scope to generate pseudo real-time 3 -D image during operation by selecting best-fitted 3-D image from 4-D image based on real-time respiratory phase monitoring. The phantom study showed that RMS position error of imaged phantom was $2.3 \mathrm{~mm}$ in case that the stroke of the motion was $50 \mathrm{~mm}$ and $N_{\phi}$ was 8 . The error is clinically acceptable level, since the typical size of tumors for treatment is about tens millimeters. Image quality and imaging time were also evaluated changing $N_{\phi}$, and we found that there are trade-offs between imaging time and image quality or position accuracy. Imaging, however, time can be reduced by changing phase partitioning method, and we succeeded to reduce imaging time by 16 percents in the phantom study. The volunteer study demonstrated that the proposed method can be applied to the real human liver. Although the imaging time of approx. 30 minutes is thought to be applicable, a same evaluation on a patient under general anesthesia, breathing with respirator, need to be performed, since histogram of motion of the target is different from free-breath.

There are two factors that control image position accuracy and image quality in Adaptive 4-D Scan: width of respiratory phase partition, $\Delta \phi$, and time lag of real-time respiratory phase monitoring and adaptive control. $\Delta \phi$ effects inconsistency in position of the phantom, where MR echo signals are acquired for each 3-D image. This inconsistency causes position error and blur/noise in images, as shown in the phantom study. The second factor, time lag, leads inaccuracy in monitoring of $\phi$ and also causes inconsistency in position of the phantom. Time lag was determined by repetition time (TR) of echo signal acquisition and throughput of the scanner system, and currently it is more than $100 \mathrm{~ms}$. In Fig 5 (1), position error is improved up to $N_{\phi}=8$, but it is almost unchanged when 
$N_{\phi}>8$. We think this is because the effect of time lag is constant for all $N_{\phi}$, while the effect of $\Delta \phi$ can be reduced by increasing $N_{\phi}$, and primary factor controlling the position error is changed from $\Delta \phi$ to time lag around $N_{\phi}=8$.

The frame rate of pseudo 3-D navigation image generated from 4-D image is theoretically limited by that of tracking and, more than $30 \mathrm{fps}$ could be achieved by using the developed system. We think that the proposed method would be suitable for automated targeting on moving organ for MRI-guided robotics [8] and focused ultrasound surgery (FUS) 9 .

In conclusion, we proposed Adaptive 4-D Scan technique for real-time intraoperative MRI. Phantom and volunteer study shows the proposed method is feasible for clinical use and has capability to provide real-time dynamic 3-D atlas for surgical navigation.

This work is partially supported by Research Fellowships of the Japan Society for the Promotion of Science (JSPS) for Young Scientists.

\section{References}

1. Blackall, J. M., King, A. P., Penney, G. P., Adam, A., Hawkes, D. J.: A statistical model of respiratory motion and deformation of the liver. Medical Image Computing and Computer-Assisted Intervention - MICCAI 2001, LNCS 2208 (2001) 1338-1340

2. Tokuda, J., Hirano, M., Tsukamoto, T., Dohi, T., Hata, N.: Integration of Projection Profile Matching into Clinical MR Scanner System for Real-time Organ Tracking and Image Registration, Medical Image Computing and Computer-Assisted Intervention - MICCAI 2003, LNCS 2879 (2003) 311-318

3. von Siebenthal, M., Cattin, P., Gamper, U., Lomax, A.,Székely G.: 4D MR Imaging Using Internal Respiratory Gating, Medical Image Computing and ComputerAssisted Intervention - MICCAI 2003, LNCS 2879 (2003) 311-318

4. Liu, Y.L., Riederer, S.J., Rossman, P.J., Grimm, R.G., Debbins, J.P., Ehman, R.L.: A monitoring, feedback, and triggering system for reproducible breath-hold MR imaging, Magnetic Resonance in Medicin 30 (1993) 507-511

5. Kolmogorov, V.N., Watts, R., Prince, M.R., Zabih, R., Wang, Y.: Simultaneous multiple volume (SMV) acquisition algorithm for real-time navigator gating, Magnetic Resonance Imaging 21 (2003) 969-975

6. Ehman, R. L., Felmlee, J. P.: Adaptive Technique for High Definition-MR Imaging of Moving Structures Navigator Echoes. Radiology 173 (1989) 255-263

7. Gering, D. T., Nabavi, A., Kikinis, R., Hata, N., O'Donnell, J., Wells III, W. M.: An Integrated Visualization System for Surgical Planning and Guidance Using Image Fusion and an Open MR. J. of Magn. Reson. Im. 13 (2001) 967-975

8. Chinzei, K., Hata, N., Jolesz, F. A., Kikinis, R.: MR compatible surgical assist robot: System integration and preliminary feasibility study. Medical Image Computing and Computer-Assisted Intervention - MICCAI 2000, LNCS 1935 (2000)921-930

9. Jolesz, F.A., Hynynen, K.: Magnetic resonance image-guided focused ultrasound surgery. Cancer J., 8 (2002) S100-S112 